\title{
Journal of Electrical Bioimpedance (JEB) - a new, open access, scientific journal
}

\author{
Sverre Grimnes and Ørjan G. Martinsen \\ 1. Editors-in-chief, Journal of Electrical Bioimpedance \\ 2. Department of Physics, University of Oslo, Oslo, Norway \\ 3. Department of Biomedical and Clinical Engineering, Rikshospitalet, Oslo University Hospital, Oslo, Norway
}

Bioimpedance is a specialized field under constant development. Perhaps more than one thousand researchers worldwide are working in this field at universities, research institutes and in the industry. The application area is wide and increasing, the scientific basis stable but also under constant development. It is now possible to exploit the possibilities opened up by the new efficient media systems enabling low cost and rapid publication. We believe that a dedicated journal on bioimpedance will stimulate the spread of better and more homogeneous research information when based on selectivity, rapidity, open (free) access, free of charge, and not to forget a specialized international peer review system with each submitted article reviewed by more than two experts.

JEB is needed both for presenting new applications and basic scientific developments. The application areas are widest in human medicine, e.g. in dermatology, cardiology, neurology, surgery, sport medicine, physiotherapy, psychophysiology and cognitive sciences. Objects of interest are patients, test persons, body and organs, and cell suspensions. Bioimpedance instrumentation is for monitoring physiological parameters, sensors, imaging systems, tissue and organ status characterization such as nutrition and body composition, ischemia, edema and cancer. Applications also extend into veterinary medicine, food research and agriculture (e.g. plant physiology), and outside medicine penetrating new application areas for cell suspensions (e.g. brewery), cell cultures and bacteria detection. Also of interest are the engineering aspects of nanotechnology electrodes, semi-invasive electrodes through the upper layers of the skin, new tissue contact electrolytic materials, and telemetry systems with a nongrounded (floating) patient. All this only to mention some of the application areas where bioimpedance is in active development.

Electrical bioimpedance, bioelectricity and electrical properties of tissue are much about the same things and therefore within the interest range of JEB. Geddes [1] defined an important application area: "Detection of physiological events by impedance". Bioimpedance has important transducing properties and can be used in all sorts of sensor systems. The strength is that many important physiological parameters can be measured, and the weakness that bioimpedance as an electrical variable does not in itself give the necessary selectivity. A scientific sobriety is necessary in this application field.

Hope of important results is also present on tissue characterization in normal and pathological tissue, discovering tissue and organ cancer and ischemia with new electrode systems, discovering lung edema and transplant organ rejection, and doing life sign monitoring with new body scanners.

Important developments in basic bioimpedance science are for instance new applications of the Maxwell equations (still not exhausted!), Helmholz reciprocity theorem, Cole models, Finite Element method (FEM), and impedance tomography imaging (EIT). There are unsolved problems in mapping current density fields, sensitivity fields and anisotropy. Modeling, dispersion mechanisms, non-linearity and cell membrane poration are subjects under development. New basic concepts suddenly emerge, e.g. the recently presented memristor component. Because of the complexity of tissue we usually do not know the detailed current paths in the volume. The basic problem of electrically characterizing tissue is the homogeneity, because it does not exist. Tissue is complex on all scales and parameters vary according to the size of the sampled volume.

As already pointed out we consider bioimpedance as a truly cross-disciplinary subject based upon physics, engineering, medicine, chemistry, material science, mathematics, computer science and more. With such a scattered foundation our discipline has always had a need for a multidisciplinary research network and we sincerely hope that JEB will serve as such. JEB represents an offer to all our serious scientists and researchers: We welcome you to the new journal!

\section{Acknowledgments}

We have looked forward to this moment when we could present a new open access scientific journal on electrical bioimpedance to the public. We are grateful to the University of Oslo for making this possible. We furthermore thank the Department of Biomedical end Clinical Engineering at Rikshospitalet and the Department of Physics at the University of Oslo for their strong support. We are also indebted to Stine-Marie Barsjø at the Oslo University Library and Morten Erlandsen at USIT for their invaluable help.

\section{References}

1. Geddes LA, Baker LE. Principles of applied biomedical instrumentation. 3. ed. Wiley; 1989. p. 537. 